\title{
GLOBAL MATCHING FOR AIR CONDITIONING INSPECTION ROBOT
}

\author{
Yasunori Abe*1, Toshio Fukuda*2, Masaru Shikano*3, \\ Fumihito Arai*3,Yoshio Tanaka*1, and Shintaro Sakamoto*1
}

\author{
*IResearch and Development Center, Shinryo Corporation \\ *2Center for Cooperative Research in Advance Science \& Technology, \\ Nagoya university \\ *3Department of Micro System Engineering, Nagoya University
}

\begin{abstract}
This paper proposes the navigation system of autonomous mobile robot. The conventional robot was moved in the navigation method based on the landmark recognition. But, the landmark was often lost sight of, as the result the robot's self-position was lost. The authors propose the method that the error that was occurred by the robot is recovered. The method can decide the self-position by using the information on the corner, windows and doors of the room.
\end{abstract}

Keywords: Autonoous Mobile Robot, Navigation System, Error Recovery, Landmark Recognition, Vision Recognition

\section{INTRODUCTION}

The authors have done the development of the air conditioning equipment inspection robot so far as a robot with integrated locomotion and manipulation [1]. This robot had a vision based navigation system. This robot could recognize the ceiling air diffusers for the air conditioner of the room by two $\mathrm{CCD}$ cameras. Then, it could autonomously move using them as the landmarks. Then, this robot could examine the air flow rate and temperature for the air conditioner from the landmark, ceiling air diffuser. The robots which use the landmark to move autonomously are being studied abundantly. But, perfect landmark recognition was not made of robot only by the simple template matching in the actual world by many disturbances. As a result, the robot did not do an accurate movement. Therefore it sometimes came off the movement course. It is necessary to solve this problem that the robot itself notices its sensing error, and has the ability which makes recovery. So, the authors have proposed the minus primitive, the map correspondence, the variable template matching, and so on, in order to increase the robustness of recognizing the landmark. But, a landmark is still sometimes lost sight of with the robot in the reality. So, it is necessary how to recognize the self-position without using the landmarks.

The authors propose the global matching as the way that a robot knows the self-position in this paper. The Global Matching uses the windows, doors, walls, corners, and so on of the room to detect it's self-position and it makes the robot revert in the course that was lost sight of. The robot can combine two self-position recognition methods that are in the local area and wide area by the control architecture proposed by authors. The local self-position is by the landmark recognition and the wide one is by the Global Matching. Then, the robot can move with making an error recovery.

Wang[9] proposed how to estimate the selfposition at the indoor from the matching rate of the model and the edge screen gotten by the image processing. However, it is impossible to decide the self-position of the robot only using the result of the edge detection, because there are few geometrical characteristics in the big square room which a robot works easily. Tonaki[8] proposed the method which the position was decided by using two laser luminous units. This method can expect satisfactory measurement precision. But, human work increases because a measurement unit must be installed precisely every time. Furthermore, Ando[6] and Oriolo[7] proposed how to detect the self-position by using the ultrasonic sensor to measure a distance from the wall. But, because the measurement range of the ultrasonic sensor is short, this method can not be measured directly in the large room. These techniques can not detect an efficient self-position in the usual big office room. So, the authors propose this technique. It is stated about the algorithm, the position measurement precision and the result of the movement experiment by this paper. 


\section{ROBOT WITH INTEGRATED LOCOMOTION AND MANIPULATION}

\subsection{Air Conditioning Equipment Inspection Robot}

We have developed the robot with integrated locomotion and manipulation that has the navigation system using the air diffusers on the ceiling as the landmarks and it is able to inspect the air flow rate and temperature pursuing the air diffusers. The ceiling air diffuser is called Anemo and it is shown in Fig.1.

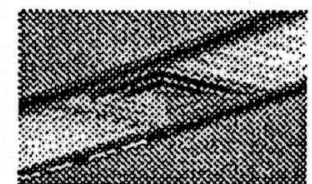

Fig.1 Anemo and System Line

The robot was equipped two CPUs and two CCD cameras. The robot consists of four sub modules; the vision system, the vehicle system, the sensor system and the lift-up system. One CPU manages the vision system that recognizes the landmarks and calculates the robot's position, and the other one manages the vehicle system, the sensor system that checks the air-conditioning and the liftup system that lifts up the sensor system to the ceiling. We show the robot in Fig.2.

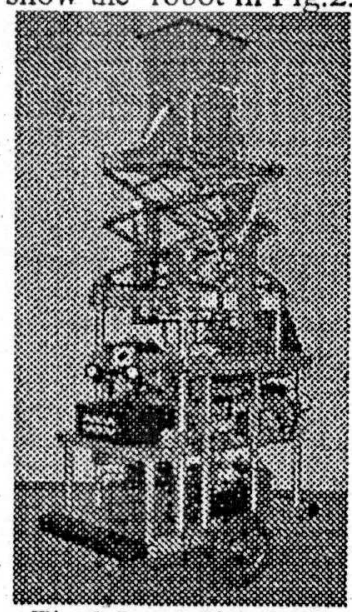

Fig.2 Inspection Robot

\subsection{Control Architecture}

A control architecture is essential to control a robot efficiently and intellectually and there are many places to learn from the creature to study the behavior of the intellectual robot. The authors propose HALAS (Hierarchical Adaptive and Learning Architecture System) as a control architecture of the navigation system of the autonomous mobile robot in consideration of the error recovery, Fig.3. The HALAS has the structure comparing the voluntary movement and the involuntary movement of vertebrate animal. The HALAS does prevention of recurrence of the error by the voluntary movement, and it can do the discovery of the error and repair by the involuntary movement. The involuntary movement is composed by some action sets by the sensing level. They are (1) Confident Action Set, (2) Discreet Action Set, (3) Reconsideration Action Set, and (4) Reconstruction Action Set. The voluntary movement can choose the action set which is suitable for the error recovery by the reinforcement learning. The wind which it can get when it reaches a landmark at last is being used as the reward of the reinforcement learning. Then the action set that has the highest evaluation function value is chosen at that time. Even if an error occurs in the landmark recognition, this robot makes recovery by using HALAS, and it can continue the work.

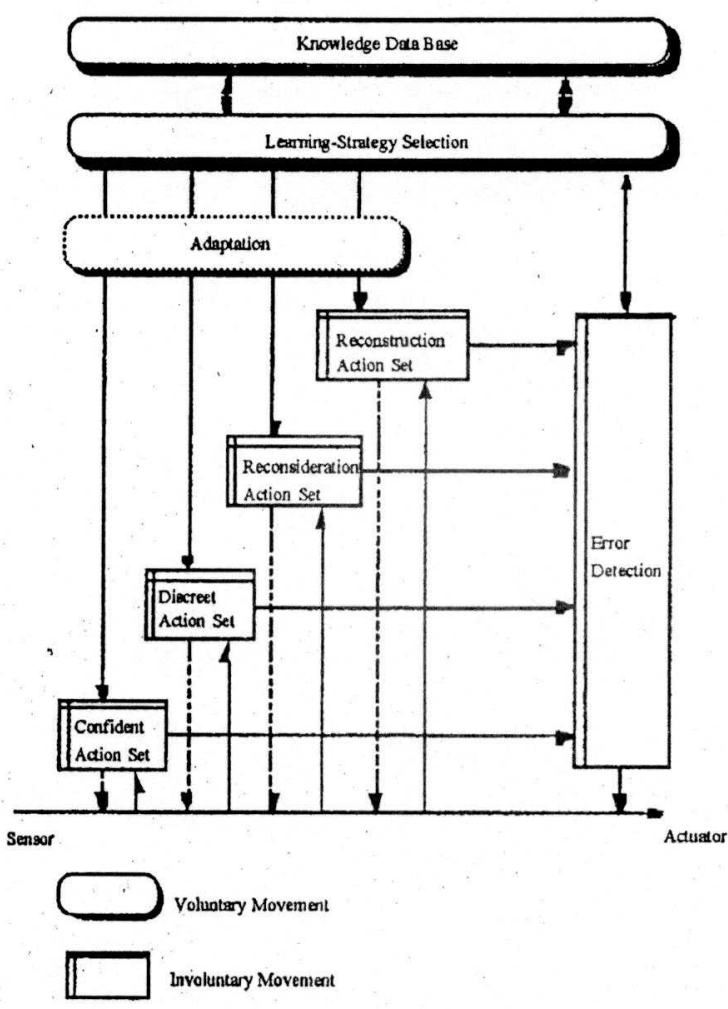

Fig. 3 HALAS

\section{LANDMARK RECOGNITION METHOD}

This robot has the following four methods as a technique of the landmark recognition. These sensing methods are delivered in the action set of the HALAS. Then, the suitable sensing behavior is chosen by the HALAS. They are stated simply here because what to state about these is not a purpose in this paper. Each reference literature shows the detail of these techniques.

\subsection{Fuzzy Template Matching}

The landmark, anemo, consists of several fins and they are the special features of this landmark. Original image from a $\mathrm{CCD}$ camera contain too much 
information for computer processing. As a result, it is necessary to have a method for taking out required image information by eliminating unnecessary information. Therefore the original image is processed through edge emphasis, smoothing, binary operation, elimination of isolated points and labeling. Consequently it becomes from gray image to binary image as shown in Fig.4 [2].

The landmarks often accompany shadows because of lighting. For this reason, an algorithm is required which is able to accurately search out a landmark even though the lines composing the landmark are thick to a certain extent. Under these circumstances, we proposed a method for expressing the templates in terms of fuzzy sets having triangular cross sections with a height of 1 as illustrated in Fig.5. The robot does a template matching in this fuzzy template. Then, the movement rule of this template can get it by a neural network. This technique is called the Fuzzy Template Matching (FTM).

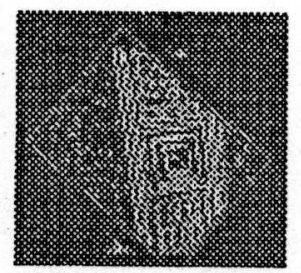

Fig.4 Binary Image

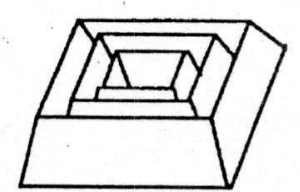

Fig.5 Fuzzy Template

\subsection{Minus Primitive}

Minus Primitive (MP) is the way of distinguishing the landmark from the resemblance things[3]. The objects are decomposed into the primitive. Then, the primitive of the anemo is defined as the plus primitive (PP), and the primitive of the resemblance thing is defined as the minus primitive(MP), and the accurate anemo recognition can be done during the actual environment by comparing a degree of each primitive in the FTM.

\subsection{Map Correspondence}

The map correspondence (MC) function makes recovery of the recognition error of the vision sensing using map information[4]. This function is called a map correspondence. The robot makes recovery of the error of the vision recognition doing the confirmation complying with of the map information and the selfposition recognition result. Furthermore the robot can find anemo with tentative movement.

\subsection{Variable Template Matching}

The variable template matching (VTM) can be done by changing the size of the template and the position suitably for the accurate anemo recognition is stated[5]. The robot makes a histogram of the binary density projection at the right after it got an outline drawing of the anemo and presumes the parameter of the size and the position of the anemo inside the image. Then, the robot uses this result as an initial individual of the genetic optimization technique, and do template matching.

\section{GLOBAL MATCHING}

The robot which lost sight of a landmark gets lost the path it should go along. So, this robot does the Global Matching, and detects its position, and reverts in the course of the base. This Global Matching Method is one of the action sets including the above mentioned (4) Reconstruction Action Set in HALAS. The Global Matching is the behavior that a robot can recognize a self-position absolutely by the coordinates system of the big room in at the any position. The self-position is shown in the twodimensional coordinates $\mathrm{x}$ and $\mathrm{y}$, and the posture angle $\theta$. In this research, the robot searches the position on the corner inside the room by the main camera, and it is confirmed. Then, the robot calculates its position with the corner.

\subsection{Environmental Condition}

The environment that the Global Matching is done, and the precondition are explained here. The environment that it is prepared for the experiment is the room of the area of $26 \mathrm{~m} \times 11 \mathrm{~m}$ which is shown in Fig.6. This is the environment which generally an air conditioning equipment inspection robot is used for. The robot has the design chart at this room. Therefore, the dimension of the room, the position and size of the anemo and the window, the position of the door, the height of the ceiling, and so on are well-known. The inspection route of the anemo is decided by a human in advance. Furthermore, other environment conditions and preconditions in this technique are shown in the following.

(1) The room is the rectangle big office room and there are system line where anemo and so on is arranged on the ceiling.

(2) There is at least one wall side that there are not a pattern, a line, and a structure thing in the room. Then, there is at least one corner where the outline is clear and it touches the wall. There can be window, door and so on in other walls.

(3) The condition that it was lost sight of the selfposition is as the following.

- When the landmark is not found even if the error recovery technique stated before is used.

- When the number of the landmarks is different from the map information. 


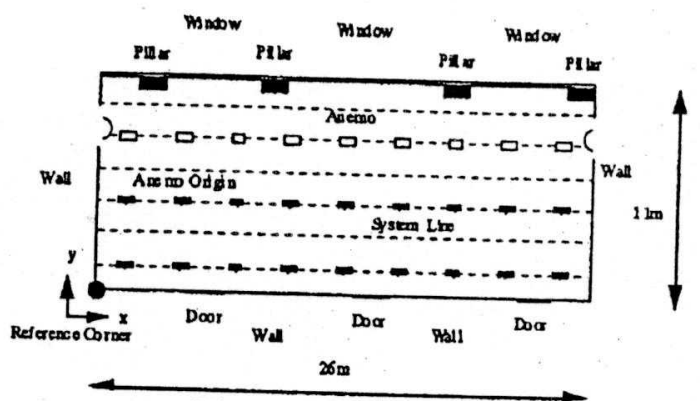

Fig.6 Experimental Room

\subsection{Orientation Modification}

To begin with this process, the robot turns to the wall for the corner detection. The robot detects the self-posture angle $\theta$ by using the system line on the ceiling. Then, the robot faces in the system line, and it makes the posture parallel to the system line. The process of the orientation modification is as the following. The robot takes pictures of the top by using the small camera which is set upright. Then, it confirms whether a system line exists. It is known from the past experiment that the small camera can find out the goal object if it is inside the range in every direction $300 \mathrm{~mm}$ from the center of the robot. But, when the robot judges that a system line does not exist in this range because a straight line is not extracted as a result of the image processing, it looks up at the ceiling with the other vision sensor, the main camera which is set on the pan-tilt table. Then, it does the search of the system line again turning the table. After that, the robot calculates the distance to the system line and the direction it, and it moves to right under the system line. The robot detects the angle of the posture of the system line with the small camera after the arrival, and it rotates to become parallel in the system line.

Table 1 Results of Orientation Modification(degree) Case(a) Result Case(b) Result

\begin{tabular}{ccccc} 
Initial & Small Camera & & & \multicolumn{2}{c}{ Main Camera } & Small Camera \\
\cline { 5 - 6 } 0 & -0.7 & & 5.3 & 0.4 \\
45 & 0.6 & & 7.7 & -0.1 \\
90 & 0.5 & 6.6 & -0.1 \\
\hline
\end{tabular}

Then, the authors did the posture compensation experiment of the robot, and confirmed the error of posture matching. The initial position of the robot in this experiment was divided into two cases. One is Case (a) Right under the system line, the other one is Case (b) Between the system line and the system line. Then, each initial posture it was inclined to $0^{\circ}, 45^{\circ}, 90^{\circ}$. Then, the robot paralleled the system line from these position and postures. The result is shown in Table 1. The orientation modification movement could be finally completed in less than $\pm 1^{\circ}$.

\subsection{Corner Detection}

The robot does the detection of the corner to calculate its position expressed by $\mathrm{x}, \mathrm{y}$ coordinate. The robot can use the corner of the ceiling side and the wall or the corner of the floor side and the wall. The corner of the ceiling side was used by this research. The images which the main camera took by the corner detection treatment are shown in order by Fig.7.

This method is explained concretely in the following. As the authors explained so far, the robot faces to the wall which a pattern is not in. First, the robot controls the pan-tilt table and the zooming of the lens to make the main camera face to the wall straightly at this position, Fig.7(a). The histogram that image pixel was projected on the $Y$ axis is made in the taken image at this time. This histogram shows the straight line which crosses the inside of the screen horizontally. The straight line which was done like is the boundary line of the wall and the ceiling side. Next, the robot controls the pan angle of the main camera so that this straight line comes in the center of the screen, Fig.7(b). Thirdly, the robot extracts the boundary line which crosses the center of the screen by Hugh change. Then, it calculates the center of gravity and adjusts the pan-tilt table so that the center of gravity comes in the center of the screen of the Fig.7(c). Fourthly, the robot detects the left end point of this straight line in this image. Then, it chases the line in the left with controlling pan-tilt table so that the end point becoming in the center of the camera again, Fig.7(d). This operation is repeated. Fifthly, the robot recognizes the straight terminal. The movement of the camera is to stop when the left end point on the boundary line comes in the center of the image, Fig.7(e). Finally, the robot confirms that there is a straight line which extends in the vertical bottom from this end point. This shows the boundary line of the wall and the wall. The angle of the pan and tilt are the directions of the corner seen from the robot.

Then, the coordinate of the corner is shown by the equations (1) and (2) in the coordinates system of Fig. 8 from the height of the ceiling side being known .

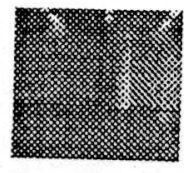

(a)

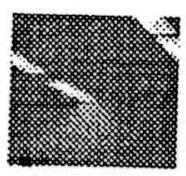

(d)

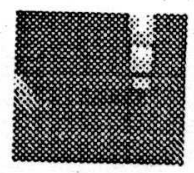

(b)

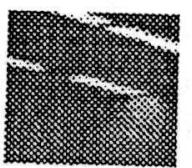

(e)

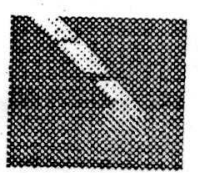

(c)
Fig.7 Image of Corner Detection 


$$
\begin{aligned}
& x_{c}=\frac{x_{v}-\cos \left(\theta_{r}+\theta_{t}-\tan ^{-1} \frac{x_{1}}{f}\right)}{\tan \left(\tan ^{-1} \frac{y_{i}}{f}+\phi_{t}\right)}\left(z_{c}-z_{v}\right) \\
& y_{c}=\frac{y_{v}-\sin \left(\theta_{r}+\theta_{t}-\tan ^{-1} \frac{x_{i}}{f}\right)}{\tan \left(\tan ^{-1} \frac{y_{i}}{f}+\phi_{t}\right)}\left(z_{c}-z_{v}\right)
\end{aligned}
$$

$x c, y c$ : The $x, y$ coordinate of the corner in the room $\mathrm{xr}, \mathrm{yr}$ : The $\mathrm{x}, \mathrm{y}$ coordinate of the robot in the room $\theta \mathrm{r}$ : The posture of the robot in the room

$\theta t, \phi t:$ The pan and tilt angle of the camera $x i, y i$ : The $x, y$ coordinate of the corner in the image $f:$ The focal distance $x v, y v, z v$ :The $x, y, z$ coordinate of the view point

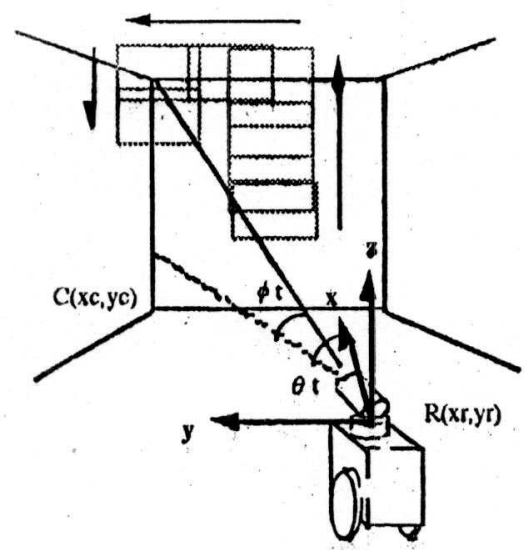

Fig. 8 Coordinates System of Corner Detection

\subsection{Evaluation of Position Error}

The authors analyzed the error. When self-position measurement is done by using this technique, five of the next is thought as a factor of the error.

(a) Inclination toward the vertical angle.

(b) The orientation modification error of the robot.

(c) The error of the comer detection by the image processing.

(d) The control error of the pan-tilt table of the main camera.

(e) Architectural error.

The influence which the error of (a) - (d) gives to the selfposition measuring was thought and the result of (a) is shown as a representative. It is shown in the maximum error circle of the self-position detection in each position inside the room in Fig. 9. But, the error circles change magnification, and it is expanded to put emphasis on the result. The data shows the diameter of the maximum error circle. The corner in the coordinate origin $(0,0)$ was detected, and the self- position was computed with the mbot in each position.

The purpose of the the Global Matching is in reverting in the movement course which deviated because an anemo was lost sight of once. Therefore, the robot should have moved from the point where the corner detection was done to the nearest anemo. However, as for the self-position measuring value, it was found out that it had a big error in the far point from the comer as the result of the error analysis. Therefore, there is area where the robot can not reach the nearby anemo directly, when the robot measures the position by only the corner detection. So, the robot moves once to the range that the precision of position measuring by the comer detection is within $\pm 300 \mathrm{~mm}$. Then, it does the self-position measuring again at the point. The anemo to approach at this time is called an origin anemo, and the robot reverts from this origin anemo to the movement course.

The robot was put on the same places where the error analysis was done on the actual office floor. Then, the self-position measuring was done at the 12 places. The result is shown as the maximum error circles in Fig. 10. The experimental result showed the tendency which was the same as the error analysis. The emor became as big as a distance from the comer was far.

From these results, it becomes clear that an origin anemo should be a $(5000,5000)$ neighborhood anemo.

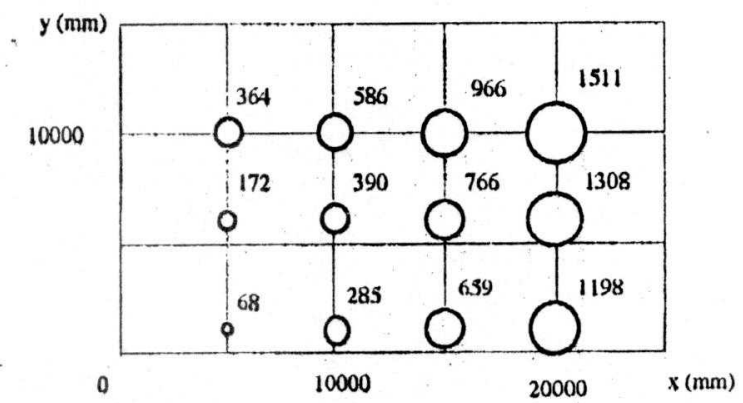

Fig. 9 Error caused by Inclination of Robot

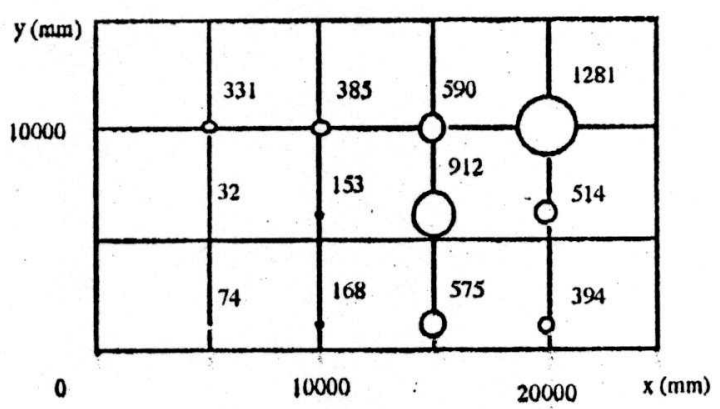

Fig.10 Experimental Result

\subsection{Topological Matching}

The coordinate of the robot based on the comer was calculated, and then the robot was moved to the origin anemo. However, it has not been confirmed yet if the robot was moved to the origin anemo or in the reverse direction. So, the robot does a final 
decision on the self-position by this technique. If the robot is in the position of Fig. 11 (a), it can decide the self-position using the map information that describes where the door and windows are. But, the door and the windows shown in the map can not be discovered if the robot faces an opposition direction in Fig. 11(b). Therefore, modification on the position is necessary. The existence of the window and the door is judged by histogram on the edge in the vertical direction in the image. The robot, recognized the window, door and wall.

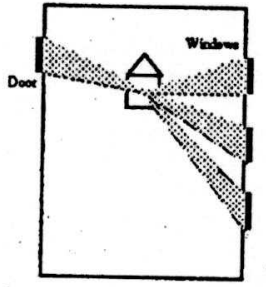

(a)

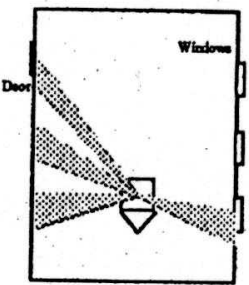

(b)
Fig.11 Topological Matching

\section{EXPERIMENTAL RESULTS OF LOCOMOTION}

The proposed technique was applicable to this robot, and the error recovery movement experiment to revert to the origin anemo from four positions was done. The movement path at that time is shown in Fig. 12. The robot was moved first with Casel and Case2 in the $y$ direction. This shows that the robot moved under the system line during the orientation modification was done. It had the self-position measurement error of $246 \mathrm{~mm}$ with Case2 by the first Global Matching movement after the orientation modification was done. The robot moved to the origin anemo $(4400,4480)$ based on this value. But, it gained the error of the dead reckoning. The error

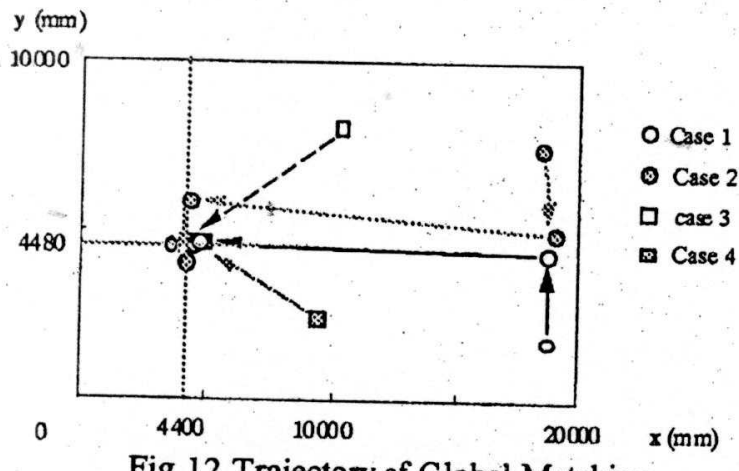

Fig.12 Trajectory of Global Matching

therefore to arrive was $1435 \mathrm{~mm}$. But, because it could approach the corner enough, the robot reduced the error by $1177 \mathrm{~mm}$ by the $2^{\text {nd }}$ Global Matching. When the robot came to this area, the anemo origin could be caught in the small camera, and the position error could be finally under $20 \mathrm{~mm}$.

\section{CONCLUSION}

It was proposed how to recognize the selfposition by the Global Matching that did not depend on the landmark, anemo. And it was an error recovery method when an anemo was lost sight of. After the robot did the orientation modification first and faced in the wall, the boundary line of the wall and the ceiling side was extracted. Then, the robot found the end of this straight line, and confirmed that there was other straight line which extended in the vertical bottom. This was confirmed as a corner. The self-position was calculated with the robot based on this corner. It confirmed that it could revert to the origin anemo by setting up around the neighborhood $(5000,5000)$ of the coordinates of the corner as the result of the experiment. Even if an anemo was lost sight of during the work and the robot deviated from the course, it was shown by this technique that the robot could revert in the movement course from the origin anemo.

\section{REFERENCES}

[1]Y.Abe, et al., "Development of Air Conditioning Equipment Inspection Robot with Vision Based Navigation System", Proceedings of the $11^{\text {th }}$ International Symposium on Automation and Robotics in Construction pp.665-674, 1994

[2]Y.Abe, et al, "Navigation System for Air Conditioning Equipment Inspection Robot", Journal of Robotics and Mechatronics, Vol.7, No.5, pp.354-366, 1995

[3]Y.Abe, et al., "Navigation System Based on Ceiling Landmark Recognition for Autonomous Mobile Robot -Position/Orientation Control by Landmark Recognition with Plus and Minus Primitives-" , Proceedings of the 1996 IEEE International Conference on Robotics and Automation, Vol.2, pp.1720-1725, 1996

[4]Y.Abe, et al., "Vision Based Navigation System Considering Error Recovery for Autonomous Mobile Robot", Proceedings of the 1997 IEEE International Conference on Robotics and Automation, Vol.3, pp.1993-1998, 1997

[5]Y.Abe, et al., "Variable Template Matching for Autonomous Mobile Robot with Hierarchical Control System", Proceedings of the Sth International Conference on Intelligent Autonomous Systems, pp.368-375, 1998

[6]Y.Ando and S.Yuta, "Following Wall by an Autonomous Mobile Robot with a Sonar-Ring", Proceedings of IEEE International Conference on Robotics and Automation, Vol.3,pp.2599-2606, 1995

[7]G.Oriolo, et al., "On-Line Map Building and Navigation for Autonom ous Mobile Robots", Proceedings of IEEE International Conference on Robotics and Automation, Vol.3, pp.2900-2906, 1995

[8]S.Tonaki and T.Takada, "Communication System of Realtime Two-dimensional Position Data with Scanning Laser Beam", Proceedings of The 13th International Symposium on Automation and Robotics in Construction pp555-560, 1996

[9]L.Wang, et al., "Model-based guidance by the longest common subsequence algorithm for indoor autonom ous vehicle navigation using com puter vision", Automation Construction, Vol. 2, No.2, pp123-137, 1993 